\title{
Tandem Nanobody: a feasible way to improve the capacity of affinity chromatography
}

\author{
Jinheng $\mathrm{Fu}^{1}$, Yunxiang Huang ${ }^{1}$, Yinfeng Zhong ${ }^{1}$, Wenyuan Shuai ${ }^{1}$, Hang Zhang ${ }^{1}$, Yanping \\ $\mathrm{Li}^{1}$, Qinghua $\mathrm{He}^{1}$, and Zhui $\mathrm{Tu}^{1}$ \\ ${ }^{1}$ Nanchang University
}

December 2, 2020

\begin{abstract}
Nanobodies, referred to the binding domain of the heavy-chain-only antibodies, are the smallest antigen recognition unit. The molecular weight of monomeric nanobodies is about one-tenth of the conventional antibodies. The small size of nanobodies facilitates genetic manipulation and recombinant expression. This study aimed to investigate the effects of nanobody multivalency on the binding capacity of affinity resin. The nanobody (namely AFV), which binds to the fragment crystallizable (Fc) region of immunoglobulin $\mathrm{G}(\mathrm{IgG})$, was fused to the N-terminal of HaloTag in the form of monomeric (H-AFV), dimer (H-diAFV), trimer (H-triAFV), and tetramer (H-tetAFV). The fusion proteins were solubly expressed in Escherichia coli yielding at least $9.9 \mathrm{mg} \mathrm{L}-1$. The biolayer interferometry confirmed an increment of avidity as the increase of AFV valences. The four recombinant proteins in crude cell lysate were site-specifically immobilized onto the Halo ligand resin via the self-labeling HaloTag, respectively. The generated affinity resins were able to isolate high purity IgG from mouse plasma. An improvement of $73.7 \%$ of the static binding capacity was achieved by the H-diAFV resin as compared to the H-AFV affinity resin.
\end{abstract}

\section{Introduction}

The variable domain of the heavy chain of the heavy-chain antibodies (VHHs), also called nanobodies, are the smallest naturally occurring antigen binding domains (Muyldermans, 2013). Because of their high stability, refoldability, and manipulable characteristics, VHHs have been developed for therapeutic (Bannas, Hambach, \& Koch-Nolte, 2017), diagnostic (Salvador, Vilaplana, \& Marco, 2019), bioimaging (Virant et al., 2018), and immunoassay (Bever et al., 2016). Efforts have been made to incorporate VHHs into biomaterials as recognition elements for affinity purification (Klooster et al., 2007; Tu et al., 2015a; Zandian \& Jungbauer, 2009).

The capacity of the adsorption resins or materials is an important feature for affinity chromatography (Kang et al., 2016). Higher adsorption capacity is a benefit to lower the cost and reduce the time for the downstream process of purification, especially in industrial applications. To increase the adsorption capacity, the key is to improve the bioactive molecular density of the recognition ligand. One approach is to use high surface-area-to-volume ratio supporting materials, such as magnetic nanoparticles (Xiong et al., 2015), porous scaffolds (Rápó et al., 2020; B. Zhang, Lalani, Cheng, Liu, \& Liu, 2011), which provide more positions for ligand coupling. Multivalent ligands provide another strategy to increase the recognition units of the affinity materials(Freiherr von Roman \& Berensmeier, 2014; Sakhnini, Pedersen, Dainiak, \& Bulow, 2020). Many biological processes, e.g. cell signaling, adhesion, are governed by multivalency (Csizmar et al., 2019). Polyvalent antibody or antibody fragment often shows better apparent binding affinity than the monomer (Rohse, Weickert, Drescher, \& Wittmann, 2020). Miller et al. demonstrated that the tetramerized domain of an anti-tumor necrosis factor antibody exhibits major increases in binding potency and in neutralizing cytotoxicity (Miller, Carr, Rabbitts, \& Ali, 2020). Zhang and co-works constructed nanobody dimmers with 3 linkers of different lengths and proved that linking nanobodies recognize distinct epitope of green 
fluorescent protein (GFP) is beneficial to purify GFP-tagged proteins (Z. Zhang, Wang, Ding, \& Hattori, 2020). Furthermore, the multivalency strategy is also proven to be effective to improve the sensitivity in immunoassay (Anderson et al., 2019; He et al., 2020).

In previous work, we developed an affinity gel for purifying immunoglobulin $G$ ( $\operatorname{Ig} G)$, which is achieved by using a self-labeling system, termed HaloTag, to orientationally immobilize the HaloTag fused anti-Fc VHH (H-AFV) onto solid support (J. Fu et al., 2019). Due to the site-specific coupling of the recognition molecule $\mathrm{H}-\mathrm{AFV}$, the static capacity was improved by $26.7 \%$ than that of randomly coupled affinity gel from $6.48 \pm 0.56 \mathrm{mg} \mathrm{mL}^{-1}$ to $8.21 \pm 0.30 \mathrm{mg} \mathrm{mL} \mathrm{mL}^{-1}$. Here, we constructed three HaloTag fused multivalent VHHs which tandemly fusing the anti-Fc VHH (AFV) in the form of bivalence (H-diAFV), trivalence (H-triAFV), and tetravalence (H-tetAFV). All fusion proteins are solubly expressed inEscherichia coli. The affinity resins were prepared using the four recombinant proteins respectively. The influence of ligand immobilizing and gel operating conditions on the capacity were investigated and compared between the four resins. Our data demonstrated that tandem VHH offers an easy way to improve the feature of affinity materials.

\section{Materials and Methods}

\subsection{Materials and reagents}

The nanobody AFV has been described previously (Tu et al., 2015b). Amine Reactive Biosensors were from Fortébio (California, USA). The Nickel Affinity Gel (NAG) and Protein A resins were from Genescript (Nanjing, China) and Sangon (Shanghai, China), respectively. Primers for polymerase chain reaction (PCR) were synthesized by Genescript (Nanjing, China). The Bradford Protein Assay Kit was purchased from Beyotime (Nanjing, China) to measure the concentration of protein solutions. The Halolink ${ }^{\mathrm{TM}}$ Resin was purchased from Promega (Beijing, China). Other reagents, salts, and buffers were of the analytical purity commercially available. The $\mathrm{pH}$ of solutions was measured by a $\mathrm{pH}$ meter (PHS-25, Leici, Shanghai, China). Ultrapure water obtained using a Milli-Q System (Millipore, USA). Empty affinity columns (1 mL) were purchased from Biocomma (Shenzhen, China).

\subsection{Vector construction, protein expression, and purification}

The expression vector pet30a-HaloTag-AFV, encoding the recombinant protein H-AFV, was described previously (Jinheng Fu et al., 2019). To construct tandem AFV expression vectors, DNA fragments encoding AFV were subcloned into the Sfi I site of pet30a-HaloTag-AFV according to standard molecular cloning protocol (Russell \& Sambrook, 2001). Briefly, the AFV encoding sequence was amplified by PCR, using the plasmid pet30a-HaloTag-AFV as a template. The primers AFV-F (5'-ata cga ctc act ata GGC CCA $G C C G G C C c$ a ggt gca gct cgt gg-3') and AFV-R (5'-TCG CGG CCG GCT GGG CC a tgg ggt ctt cgc $\left.\operatorname{tg}-3^{\prime}\right)$ were specific to the sequence of AFV. The underlined sequences at the 5' flank of the primers are the recognition site of $S f i$ I. The PCR product was digested by the restriction enzymeSfi I, gel purified and inserted into the $S f i$ I site of pet30a-HaloTag-AFV. The recombinant vectors pet30a-HaloTag-diAFV, pet30a-HaloTag-triAFV, and pet30a-HaloTag-tetAFV are encoding two, three, and four tandem AFVs, respectively. The sequences of all the expression vectors were confirmed by Sanger DNA sequencing. The recombinant vectors were transformed into $E$. coli BL21 (DE3) pLysS competent cells, respectively. A single colony was inoculated in $2 \mathrm{xYT}$ medium (containing $50 \mathrm{\mu g} / \mathrm{ml}$ kanamycin), shaking at $37{ }^{\circ} \mathrm{C}$ overnight. The culture was used to inoculate $50 \mathrm{~mL} 2 \times \mathrm{YT}$ medium containing $50 \mu \mathrm{g} / \mathrm{ml}$ kanamycin and incubated at $37^{\circ} \mathrm{C}$, $220 \mathrm{rpm}$ until the optical density at $600 \mathrm{~nm}\left(\mathrm{OD}_{600}\right)$ reached 0.5 (about $3 \mathrm{~h}$ ). To induce the expression of protein, isopropyl $\beta$-D-1-thiogalactopyranoside (IPTG) was added at a final concentration of $0.5 \mathrm{mM}$. After cultivation at $18{ }^{\circ} \mathrm{C}$ for $10 \mathrm{~h}$, the bacteria cells were harvested by centrifugation at $5000 \mathrm{~g}$ for $20 \mathrm{~min}$ and resuspended in $10 \mathrm{~mL}$ ice-cold phosphate-buffered saline (PBS, pH 7.4). The suspended cells were disrupted in an ice bath using an ultrasonic crusher (Scientz-IID, Ningbo, China). The crude cell lyse was centrifuged at $7000 \mathrm{~g}$ for $15 \mathrm{~min}$ at $4{ }^{\circ} \mathrm{C}$. The target protein in the supernatant portion was purified by NAG and analyzed by denaturing sodium dodecyl sulfate polyacrylamide gel electrophoresis (SDS-PAGE), as described previously (J. Fu et al., 2019). Protein concentrations were assessed using the Bradford Protein Assay Kit following the manufacturer's instruction. 


\subsection{Biolayer interferometry}

The purified four recombinant proteins, H-AFV, H-diAFV, H-triAFV, and H-tetAFV were diluted with PBS at the final concentration of $25.00,32.60,40.15$, and $47.70 \mu \mathrm{g} \mathrm{mL} \mathrm{L}^{-1}$, and then immobilized onto the EDC/NHS pre-activated AR2G biosensors (Sartorius AG), respectively. After a 600-seconds quench with ethanolamine and a 60-seconds wash step with PBS, biosensor tips were immersed into the wells containing mouse IgGs of serial dilutions and allowed to associate for 300 seconds, followed by a dissociation step of 120 seconds. The equilibrium dissociation constant $\left(\mathrm{K}_{\mathrm{D}}\right)$ was calculated using a 1:1 binding model in Data Analysis Software BLItzPro 1 (Sartorius AG) (Tobias \& Kumaraswamy, 2019).

\subsection{Immobilization of $H$-AFV, H-diAFV, H-triAFV, and H-tetAFV}

The four HaloTag fused recombinant proteins, H-AFV, H-diAFV, H-triAFV, and H-tetAFV, were coupled onto the Halolink ${ }^{\mathrm{TM}}$ Resin following the manufacturer's instruction, respectively. The acquired resin was stored in $20 \%$ ethanol at $4{ }^{\circ} \mathrm{C}$ after washing with PBS (containing $1 \mathrm{mg} \mathrm{mL}^{-1}$ Bovine Serum Albumin(BSA), $\mathrm{pH}$ 7.6). The coupling rate $\left(\mathrm{CR}, \mathrm{mg} \mathrm{mL}^{-1}\right)$ which represents the mass of target protein on one-milliliter resin is calculated as described previously (J. Fu et al., 2019).

\subsection{Purification of IgGs using the affinity resins}

The IgG purification assay was performed in chromatography columns according to previous work (J. Fu et al., 2019). The static capacity of the resins was determined by the batch experiment as described previously (J. Fu et al., 2019). SDS-PAGE was employed to evaluate the proteins in the pre-load sample, flow-through fraction, wash fraction, and elution.

To test the reusability of the four resins, IgG purifications were performed for 10 cycles according to the chromatography column protocol. The columns were washed successively with 20 gel volumes of Glycine-HCl buffer (0.1 M, pH 2.2) and balanced with 20 gel volumes of PBS (pH 7.4) before the next IgG purification cycle. All experiments were performed in triplicate.

\section{Results and Discussion}

\subsection{Expression and purification of recombinant proteins}

In accordance with previous work, H-AFV was expressed in E. colias a soluble protein (Fig. 1, lane 1). The other three recombinant proteins, $\mathrm{H}$-diAFV, $\mathrm{H}$-triAFV, and $\mathrm{H}$-tetAFV, were also soluble expressed in E. coli (Table 1). The target proteins were purified by the NAG and then subjected to the SDS-PAGE analysis. The expected bands of the four purified proteins are shown at 49.0, 63.9, 78.6, and 93.5 KDa, respectively (Fig.1).

\subsection{Kinetic and affinity measurements}

The biolayer interferometry was used to measure the molecular interaction between recombinant proteins and the mouse IgGs, respectively. The four proteins were immobilized onto the sensor under equal molar concentration (Fig. 2). The data shows that the apparent affinity, or avidity, was improved from $77.3 \mathrm{nM}$ to $41.8 \mathrm{nM}$, as the number of antigen binding domains increased (Table 2). The tetramer H-tetAFV exhibits the highest avidity of $41.8 \mathrm{nM}$. Kinetic analysis confirms the enhancement of avidity is mainly contributed by the ascending of the association constant $\left(\mathrm{K}_{\mathrm{on}}\right)$.

\subsection{Capture recombinant proteins directly from cell lysate}

The HaloTag, which enables forming a covalent bond between the Halo ligand and the amino acid residue, is fused to the $\mathrm{C}$ terminal of $\mathrm{AFV}$ or tandem AFVs. Previous work demonstrated that the monovalence protein $\mathrm{H}-\mathrm{AFV}$ can be efficiently immobilized on the agarose bead directly from the supernatant of cell lysate (Jinheng Fu et al., 2019). To test whether the multivalency of AFVs would affect the specificity of HaloTag or not, the supernatant of each cell lysate was mixed with Halolink ${ }^{\mathrm{TM}}$ Resin. The SDS-PAGE shows that the bands of recombinant proteins were dramatically decreased after the coupling reaction (Fig. 3). The data demonstrate that the HaloTag fused tandem AFVs are specifically immobilized onto the resin as the 
same as monomeric H-AFV. The one-step immobilized resins were used in the following experiment for IgG purification of mouse plasma. The result shows that IgGs were specifically separated by the direct-coupled resins, which confirms the immobilized tandem AFVs are capable to recognize IgGs (Fig. 4).

\subsection{Effect of the coupling rate on the binding capacity for IgG}

To improve the static binding capacity for $\operatorname{IgG}$, the input of recombinant proteins were optimized in the conjugation reaction. The coupling rate for all four resins increased significantly as the increments of the recombinant proteins (Fig. 5). However, the static binding capacity of $\mathrm{H}-\mathrm{AFV}, \mathrm{H}$-diAFV, and $\mathrm{H}$-tetAFV resins was decreased when more than $8 \mathrm{mg}$ of recombinant proteins per $\mathrm{mL}$ resin was adopted in the coupling reaction. For the H-triAFV resin, the binding capacity became steady when the input H-triAFV was greater than $5 \mathrm{mg} \mathrm{mL}^{-1}$.

Unexpectedly, the increased number of AFV is not associated with an increase in the static capacity. The IgG static capacities of H-AFV, H-diAFV, H-triAFV, and H-tetAFV resin were 7.53+-0.41, 13.08+-0.59, $7.50+-0.86$, and $12.76+-0.66 \mathrm{mg} \mathrm{mL}^{-1}$ at the optimized coupling rate (Table 3). The static capacities of $\mathrm{H}$-diAFV and H-tetAFV resin were improved by $73.7 \%$ and $69.5 \%$ than that of H-AFV resin, whereas the $\mathrm{H}$-triAFV resin was similar to the $\mathrm{H}-\mathrm{AFV}$ resin. The mole ratio of IgG and the coupling ligand on the resin was calculated to evaluate the capture efficiency of immobilized recombinant proteins (Table 3). The mole ratio for all four resins was lower than their corresponding count of binding domains, indicating an unavailable of recognition domain(s) which might be due to space steric effect.

\subsection{Reusability of tandem AFV resins}

The H-diAFV and H-tetAFV resins were subjected to recycling tests since they show higher binding capacity than the monomer $\mathrm{H}-\mathrm{AFV}$ resin. The static binding capacity of both resins remains above $75 \%$ after reused 10 times (Fig.6). The capacity loss slows down after the fifth recycling, which indicates the stability of tandem AFV resins.

\section{Conclusions}

Nanobody offers several attractive properties such as more soluble and stable than canonical antibodies. This study takes advantage of the small size of nanobodies to make tandem nanobodies as the capture units of affinity resin. The tandem nanobodies are solubly expressed inE. coli and can be covalently immobilized onto the Halo ligand resin directly from crude cell lysate without pre-purification. The strategy of nanobody multivalency provided a significant improvement in the static binding capacity of the affinity gel, which is adaptive to a variety of affinity chromatography technologies.

\section{Conflict of interest statement}

The authors declare no conflict of interest.

\section{Acknowledgments}

This work was supported by the National Key R\&D Program of China (Grants 2018YFC1602203), the National Natural Science Foundation of China (No. 31860260), the Science and Technology Innovation Platform Project of Jiangxi Province (No. 20192BCD40001), and the Research Program of State Key Laboratory of Food Science and Technology (No. SKLF-ZZA-201912).

\section{References}

Anderson, G. P., Liu, J. L., Shriver-Lake, L. C., Zabetakis, D., Sugiharto, V. A., Chen, H. W., . . . Goldman, E. R. (2019). Oriented Immobilization of Single-Domain Antibodies Using SpyTag/SpyCatcher Yields Improved Limits of Detection. Anal Chem, 91 (15), 9424-9429. doi:10.1021/acs.analchem.9b02096

Bannas, P., Hambach, J., \& Koch-Nolte, F. (2017). Nanobodies and Nanobody-Based Human Heavy Chain Antibodies As Antitumor Therapeutics.Frontiers in Immunology, 8 . doi:10.3389/fimmu.2017.01603 
Bever, C. S., Dong, J. X., Vasylieva, N., Barnych, B., Cui, Y., Xu, Z. L., . . . Gee, S. J. (2016). VHH antibodies: emerging reagents for the analysis of environmental chemicals. Anal Bioanal Chem . doi:10.1007/s00216-016-9585-x

Csizmar, C. M., Petersburg, J. R., Perry, T. J., Rozumalski, L., Hackel, B. J., \& Wagner, C. R. (2019). Multivalent Ligand Binding to Cell Membrane Antigens: Defining the Interplay of Affinity, Valency, and Expression Density. Journal of the American Chemical Society, 141 (1), 251-261. doi:10.1021/jacs.8b09198

Freiherr von Roman, M., \& Berensmeier, S. (2014). Improving the binding capacities of protein A chromatographic materials by means of ligand polymerization. $J$ Chromatogr A, 1347, 80-86. doi:10.1016/j.chroma.2014.04.063

Fu, J., Li, J., Wang, W., Wu, H., Zhou, P., Li, Y., . . . Tu, Z. (2019). One-step orientated immobilization of nanobodies and its application for immunoglobulin purification. J Chromatogr A, 1603, 15-22. doi:10.1016/j.chroma.2019.06.028

Fu, J., Li, J., Wang, W., Wu, H., Zhou, P., Li, Y., . . . Tu, Z. (2019). One-step orientated immobilization of nanobodies and its application for immunoglobulin purification. Journal of chromatography. A, 1603, 15-22. doi:10.1016/j.chroma.2019.06.028

He, J. X., Ma, S. J., Wu, S., Xu, J. J., Tian, J. S., Li, J., . . . Xu, T. (2020). Construction of Immunomagnetic Particles with High Stability in Stringent Conditions by Site-Directed Immobilization of Multivalent Nanobodies onto Bacterial Magnetic Particles for the Environmental Detection of Tetrabromobisphenol-A. Analytical Chemistry, 92 (1), 1114-1121. doi:10.1021/acs.analchem.9b04177

Kang, H. J., Choe, W., Min, J.-K., Lee, Y.-m., Kim, B. M., \& Chung, S. J. (2016). Cyclic peptide ligand with high binding capacity for affinity purification of immunoglobulin G. Journal of Chromatography A, 1466 , 105-112. doi:10.1016/j.chroma.2016.09.007

Klooster, R., Maassen, B. T., Stam, J. C., Hermans, P. W., Ten Haaft, M. R., Detmers, F. J., . . . Theo Verrips, C. (2007). Improved anti-IgG and HSA affinity ligands: clinical application of VHH antibody technology.Journal of Immunological Methods, 324 (1-2), 1-12. doi:10.1016/j.jim.2007.04.005

Miller, A., Carr, S., Rabbitts, T., \& Ali, H. (2020). Multimeric antibodies with increased valency surpassing functional affinity and potency thresholds using novel formats. mAbs, 12 (1). doi:10.1080/19420862.2020.1752529

Muyldermans, S. (2013). Nanobodies: natural single-domain antibodies.Annu Rev Biochem, 82 , 775-797. doi:10.1146/annurev-biochem-063011-092449

Rapo, E., Aradi, L. E., Szabo, A., Posta, K., Szep, R., \& Tonk, S. (2020). Adsorption of Remazol Brilliant Violet-5R Textile Dye from Aqueous Solutions by Using Eggshell Waste Biosorbent. Scientific reports, 10 (1), 8385. doi:10.1038/s41598-020-65334-0

Rohse, P., Weickert, S., Drescher, M., \& Wittmann, V. (2020). Precipitation-free high-affinity multivalent binding by inline lectin ligands. Chemical Science, 11 (20), 5227-5237. doi:10.1039/d0sc01744b

Russell, D. W., \& Sambrook, J. (2001). Molecular cloning: a laboratory manual (Vol. 1): Cold Spring Harbor Laboratory Cold Spring Harbor, NY.

Sakhnini, L. I., Pedersen, A. K., Dainiak, M. B., \& Bulow, L. (2020). Multimeric fusion single-chain variable fragments as potential novel high-capacity ligands. FEBS Open Bio, 10 (4), 507-514. doi:10.1002/22115463.12789

Salvador, J. P., Vilaplana, L., \& Marco, M. P. (2019). Nanobody: outstanding features for diagnostic and therapeutic applications.Anal Bioanal Chem, 411 (9), 1703-1713. doi:10.1007/s00216-019-01633-4

Tobias, R., \& Kumaraswamy, S. (2019). Biomolecular Binding Kinetics Assays on the Octet Platform. In Fortebio (Ed.), Application Note 14 . 
Tu, Z., Xu, Y., Fu, J., Huang, Z., Wang, Y., Liu, B., \& Tao, Y. (2015a). Preparation and characterization of novel IgG affinity resin coupling anti-Fc camelid single-domain antibodies. Journal of Chromatography BAnalytical Technologies in the Biomedical and Life Sciences, 983 , 26-31. doi:10.1016/j.jchromb.2014.12.031

Tu, Z., Xu, Y., Fu, J., Huang, Z., Wang, Y., Liu, B., \& Tao, Y. (2015b). Preparation and characterization of novel IgG affinity resin coupling anti-Fc camelid single-domain antibodies. Journal of chromatography. B, Analytical technologies in the biomedical and life sciences, 983-984 , 26-31. doi:10.1016/j.jchromb.2014.12.031

Virant, D., Traenkle, B., Maier, J., Kaiser, P. D., Bodenhofer, M., Schmees, C., . . . Rothbauer, U. (2018). A peptide tag-specific nanobody enables high-quality labeling for dSTORM imaging. Nature Communications, 9. doi:10.1038/s41467-018-03191-2

Xiong, Y., Tu, Z., Huang, X., Xie, B., Xiong, Y., \& Xu, Y. (2015). Magnetic beads carrying poly(acrylic acid) brushes as "nanobody containers" for immunoaffinity purification of aflatoxin B1 from corn samples. RSC Advances, 5 (94), 77380-77387. doi:10.1039/C5RA15843E

Zandian, M., \& Jungbauer, A. (2009). Engineering properties of a camelid antibody affinity sorbent for Immunoglobulin G purification. Journal of Chromatography A, 1216 (29), 5548-5556. doi:10.1016/j.chroma.2009.05.051

Zhang, B., Lalani, R., Cheng, F., Liu, Q., \& Liu, L. (2011). Dual-functional electrospun poly(2-hydroxyethyl methacrylate).Journal of biomedical materials research. Part A, 99 (3), 455-466. doi:10.1002/jbm.a.33205

Zhang, Z., Wang, Y., Ding, Y., \& Hattori, M. (2020). Structure-based engineering of anti-GFP nanobody tandems as ultra-high-affinity reagents for purification. Scientific reports, 10 (1), 6239-6239. doi:10.1038/s41598-020-62606-7

Table 1. Construct and yield of recombinant proteins

\begin{tabular}{llll}
\hline Construct name & Construct description & Yield $\left(\mathrm{mg} \mathrm{L}^{-1}\right)$ & Expression Vector \\
\hline H-AFV & Single AFV with HaloTag & 10.80 & pET30a-HaloTag-AFV \\
H-diAFV & AFV dimer with HaloTag & 14.85 & pet30a-HaloTag-diAFV \\
H-triAFV & AFV trimer with HaloTag & 9.90 & pet30a-HaloTag-triAFV \\
H-tetAFV & AFV tetramer with HaloTag & 11.25 & pet30a-HaloTag-tetAFV \\
\hline
\end{tabular}

Table 2. Kinetic parameters analysis of recombinant proteins.

\begin{tabular}{llll}
\hline & $\begin{array}{l}\text { Association Constant } \\
\left(\mathrm{K}_{\mathrm{on}}, \mathrm{M}^{-1} \mathrm{~s}^{-1}\right)\end{array}$ & $\begin{array}{l}\text { Dissociation Constant } \\
\left(\mathrm{K}_{\mathrm{off}}, \mathrm{s}^{-1}\right)\end{array}$ & $\begin{array}{l}\text { Equilibrium dissociation } \\
\text { constant }\left(\mathrm{K}_{\mathrm{D}}, \mathrm{nM}\right)\end{array}$ \\
\hline H-AFV & $1.53 \times 10^{4}$ & $1.18 \times 10^{-3}$ & 77.3 \\
H-diAFV & $2.37 \times 10^{4}$ & $1.4 \times 10^{-3}$ & 59.2 \\
H-triAFV & $1.94 \times 10^{4}$ & $9.77 \times 10^{-4}$ & 50.4 \\
H-tetAFV & $2.71 \times 10^{4}$ & $1.13 \times 10^{-3}$ & 41.8 \\
\hline
\end{tabular}

Table 3. The comparison of AFV-based resins.

\begin{tabular}{|c|c|c|c|c|c|c|}
\hline Proteins & $\begin{array}{l}\text { Molecular } \\
\text { weight (KDa) }\end{array}$ & $\begin{array}{l}\text { Molecular } \\
\text { weight (KDa) }\end{array}$ & $\begin{array}{l}\text { Count of } \\
\text { binding } \\
\text { domain(s) }\end{array}$ & $\begin{array}{l}\text { Coupling rate } \\
\left(\mathrm{mg} \mathrm{mL}^{-1}\right)\end{array}$ & $\begin{array}{l}\text { IgG static } \\
\text { capacity }(\mathrm{mg} \\
\left.\mathrm{mL}^{-1}, \mathrm{n}=3\right)\end{array}$ & $\begin{array}{l}\text { Mol racial of } \\
\text { IgG/Ligand }\end{array}$ \\
\hline $\mathrm{H}-\mathrm{AFV}$ & $\mathrm{H}-\mathrm{AFV}$ & 49.0 & 1 & 5.63 & $7.53 \pm 0.41$ & 0.446 \\
\hline H-diAFV & H-diAFV & 63.9 & 2 & 3.53 & $13.08 \pm 0.59$ & 1.58 \\
\hline
\end{tabular}




\begin{tabular}{lllllll}
\hline & Molecular & Molecular & $\begin{array}{l}\text { Count of } \\
\text { beinding } \\
\text { domain(s) }\end{array}$ & $\begin{array}{l}\text { Coupling rate } \\
\left(\mathrm{mg} \mathrm{mL} \mathrm{mL}^{-1}\right)\end{array}$ & $\begin{array}{l}\text { IgG static } \\
\text { capacity }(\mathrm{mg} \\
\left.\mathrm{mL}^{-1}, \mathrm{n}=3\right)\end{array}$ & $\begin{array}{l}\text { Mol racial of } \\
\text { IgG/Ligand }\end{array}$ \\
\hline H-triAFV & H-triAFV & 78.7 & 3 & 4.26 & $7.50 \pm 0.86$ & 0.915 \\
H-tetAFV & H-tetAFV & 93.5 & 4 & 5.11 & $12.76 \pm 0.66$ & 1.202 \\
\hline
\end{tabular}

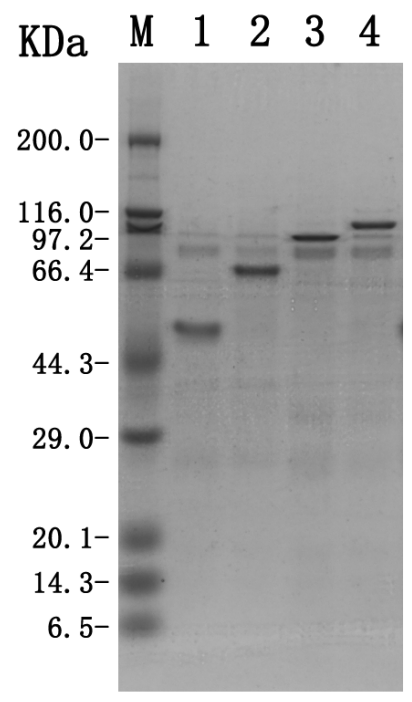



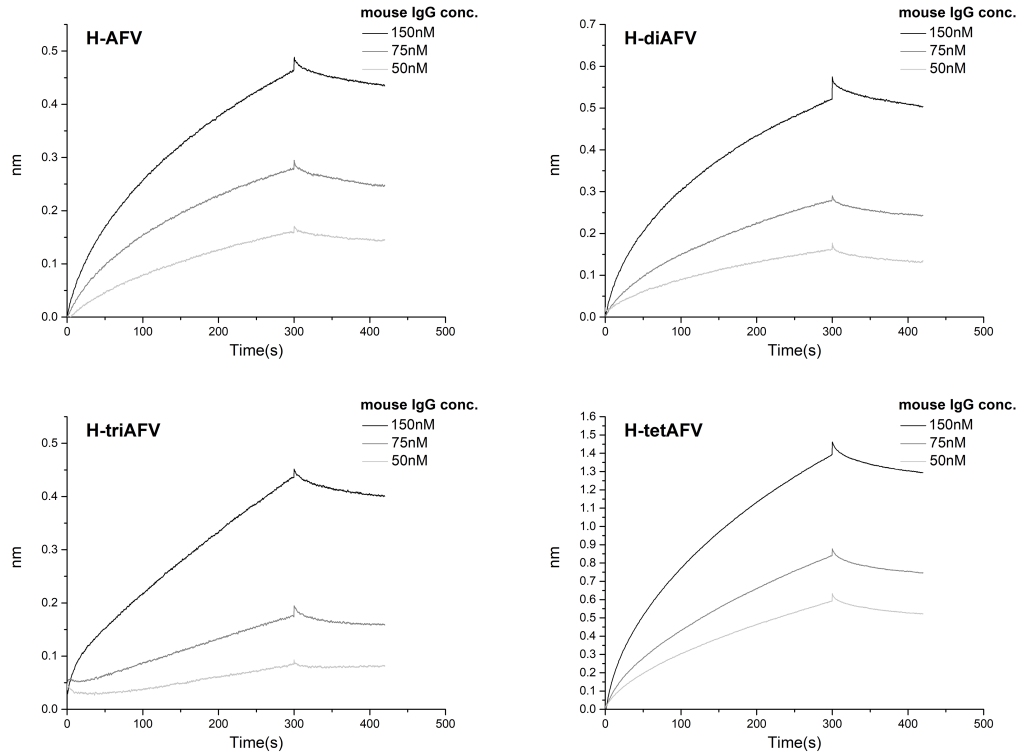

8 

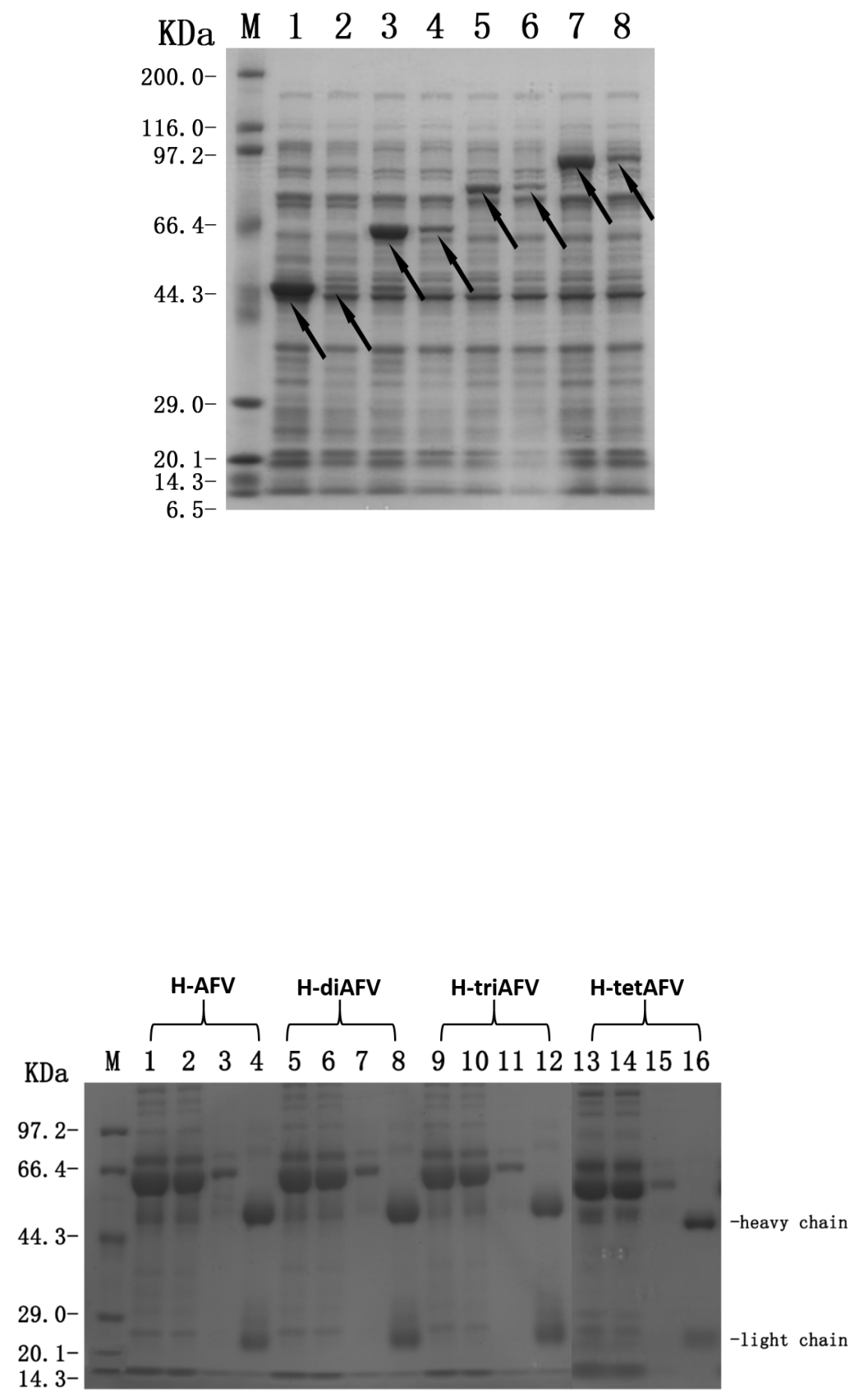

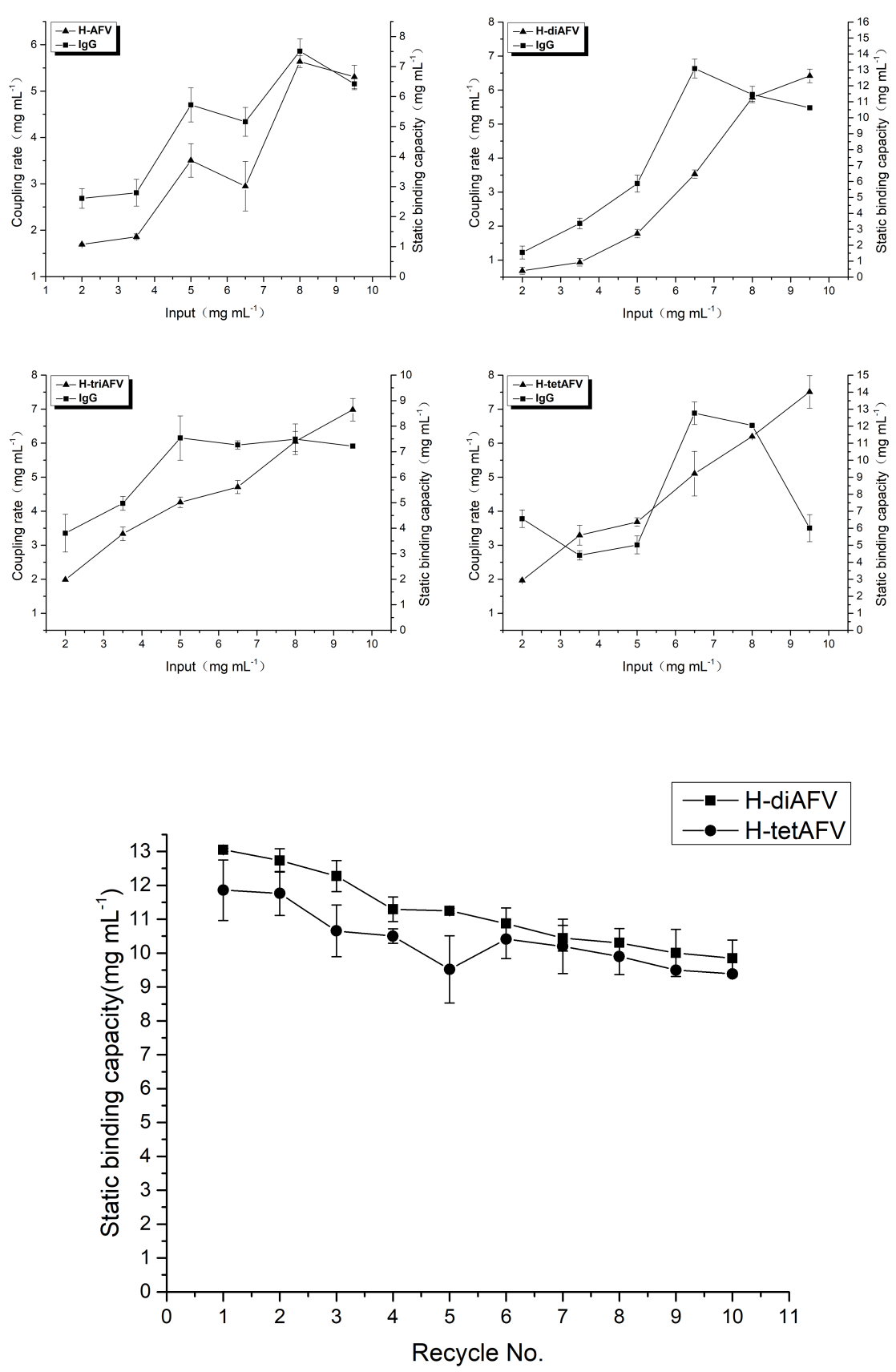